الاستقرارية الوراثية لأصناف وسلالات من الحمص Cicer arietinum L. تحت الظروف الديمية في

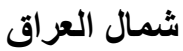

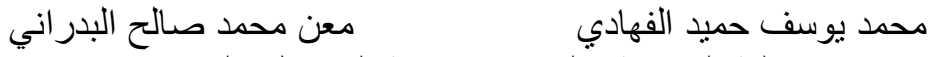 \\ كلية الزر اعة والغابات /جامعة الموصل /العراق
}

\begin{abstract}
الخلاصة

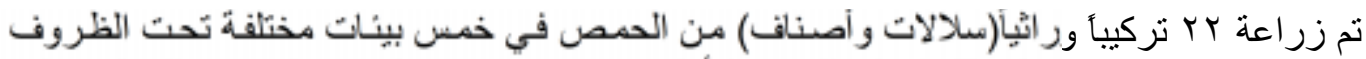

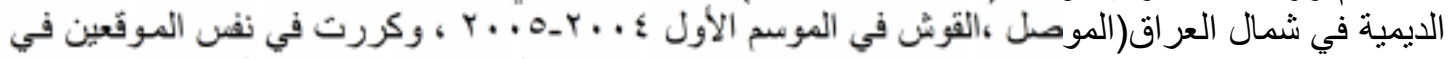

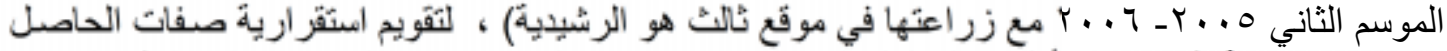

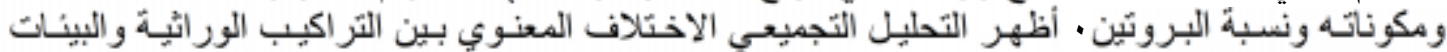

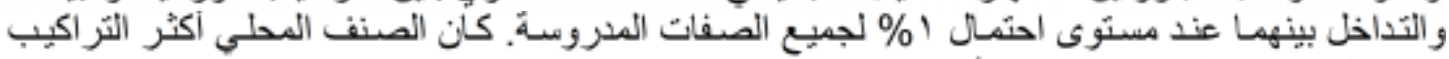

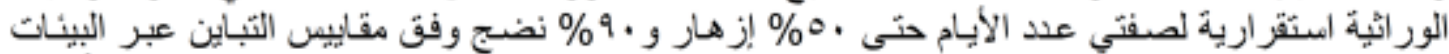

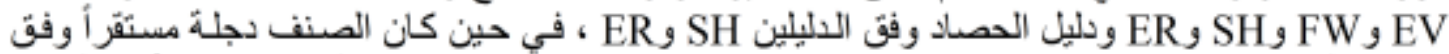

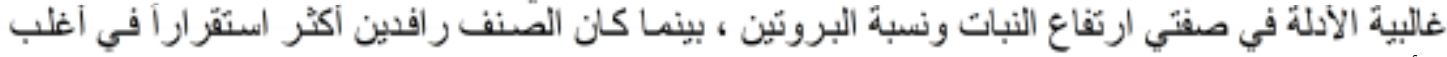

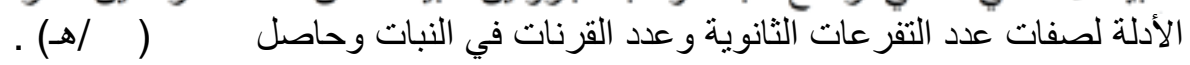

المقدمة
\end{abstract}

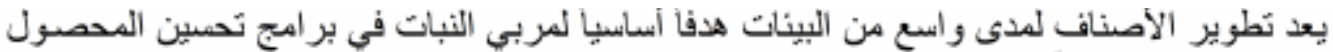

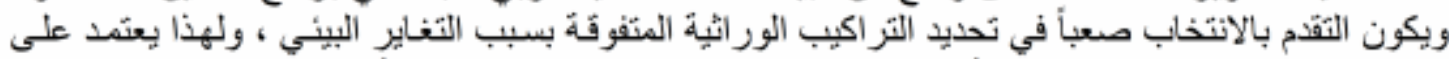

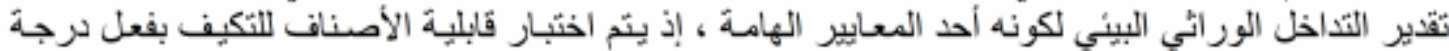

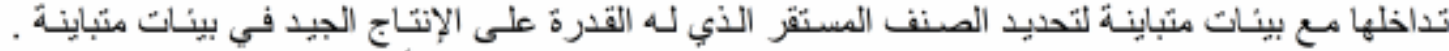

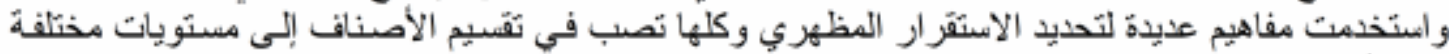

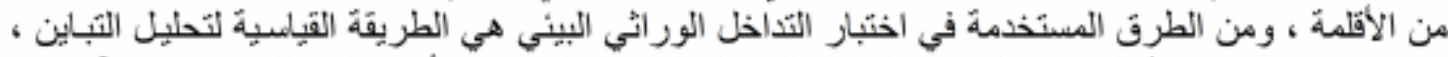

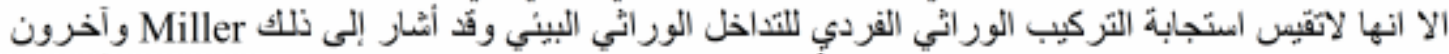

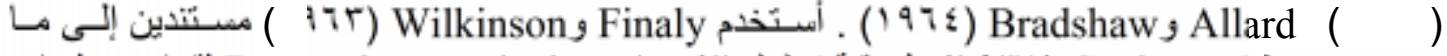

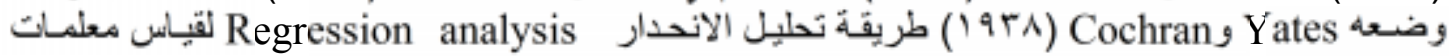

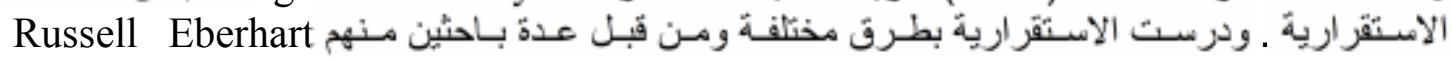

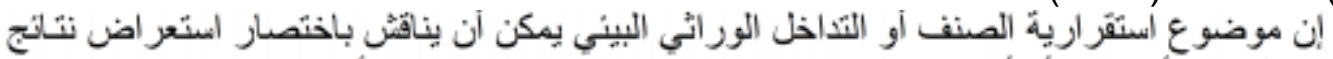

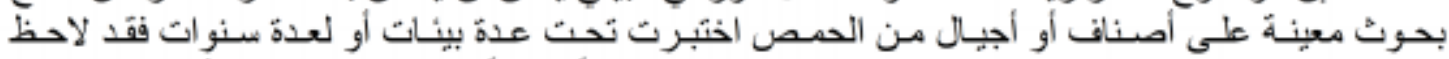
Malhotra

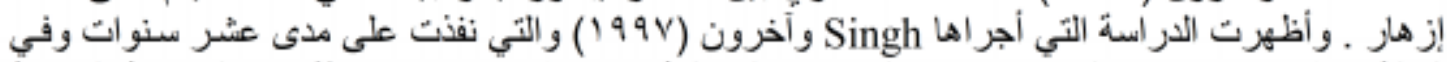

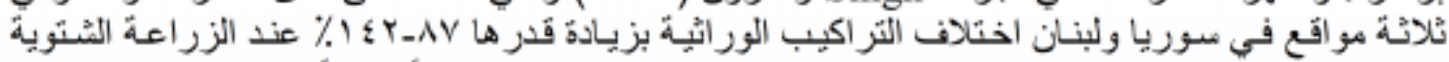

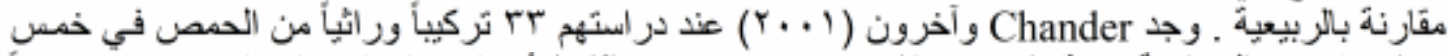

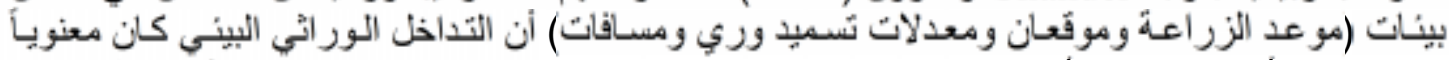

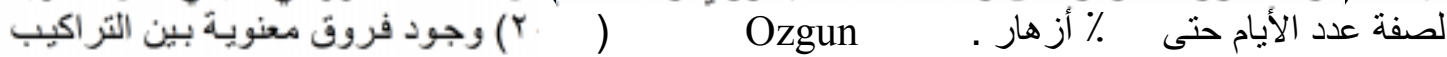

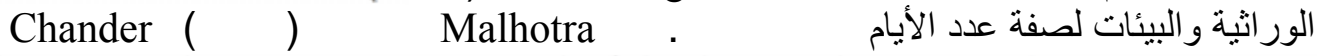

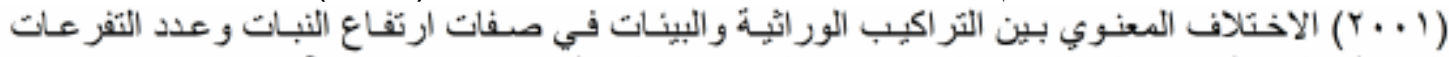

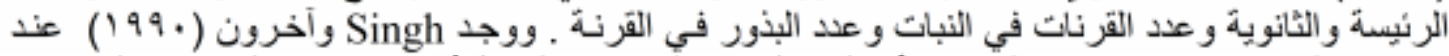
لر استهم لـ ل

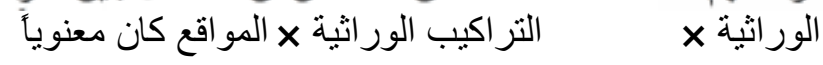


Gehla

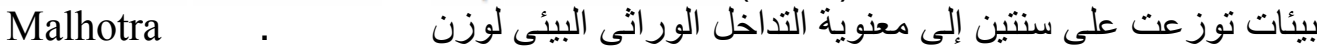

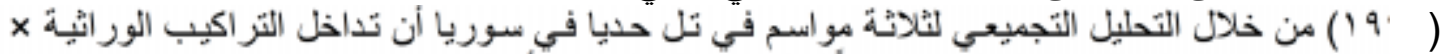

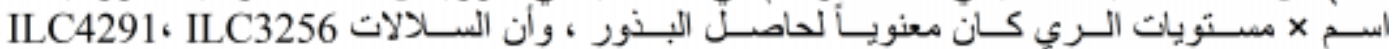

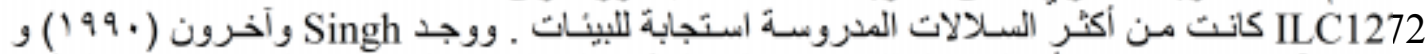

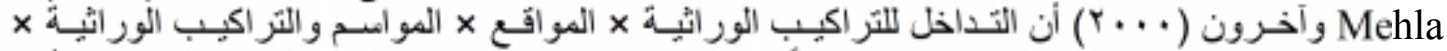

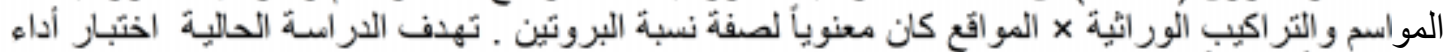

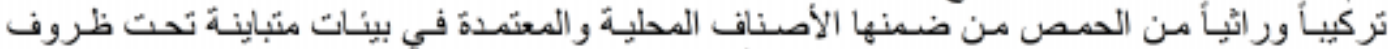
الزر اعة الديمية لتحديد ثبات الحاصل وكنو مكناته وفق أدلة مختلفة من الاستقر ارية.

\section{مواد البحث وطرائقه}

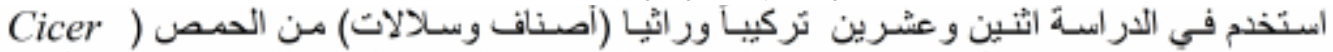

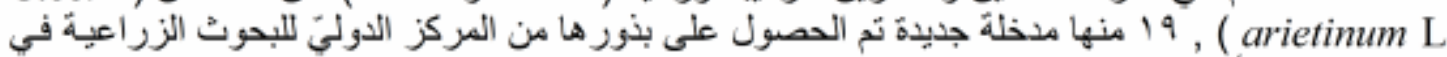

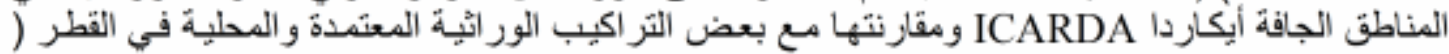

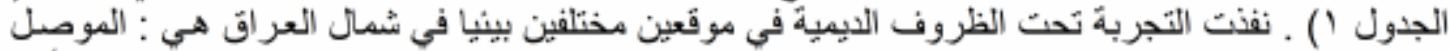

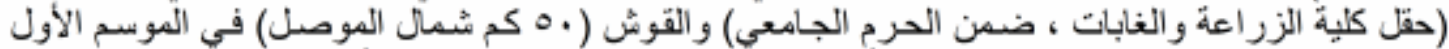

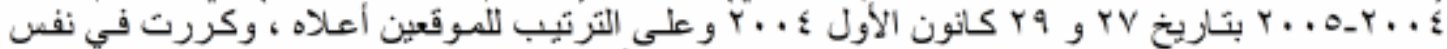

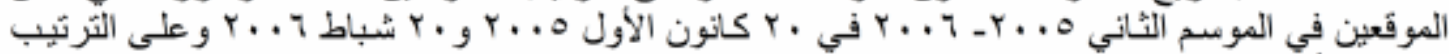

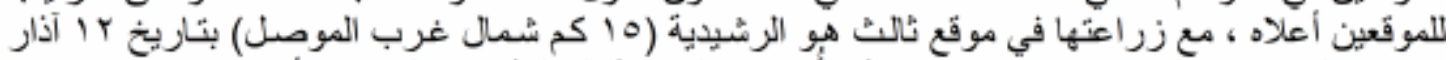

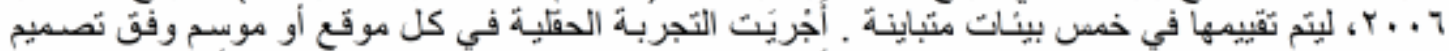

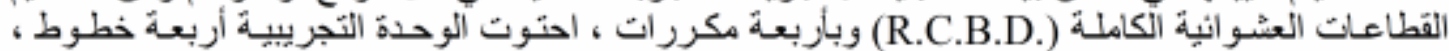

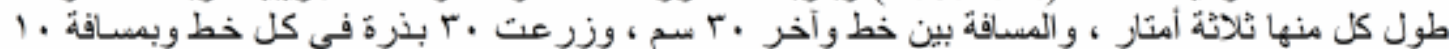

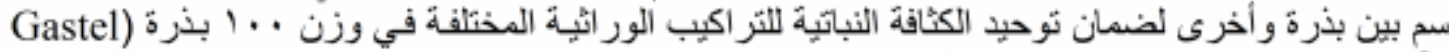

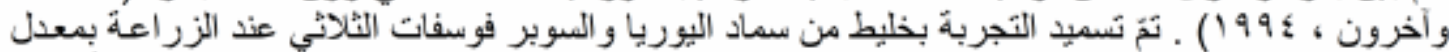

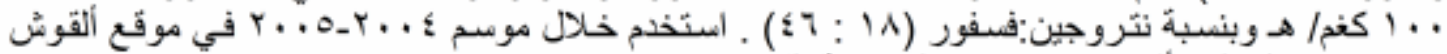

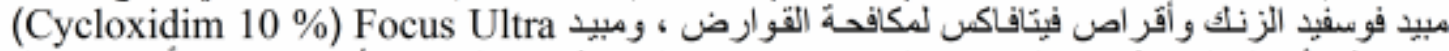

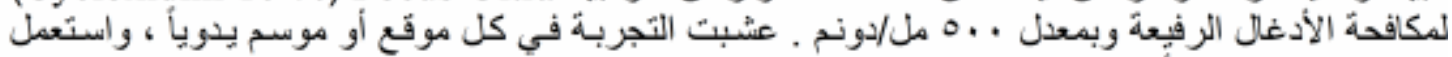
/

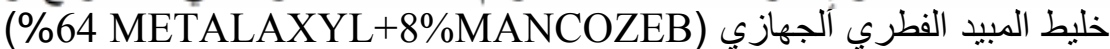

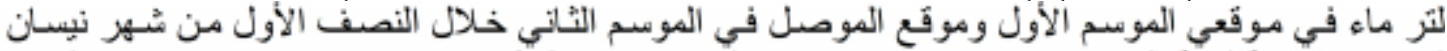
لغرض مكافحة لفحة الحمص Ascochyta blight المثّبب عن الفطر (Ascochyta rabiei ) وتم الرش

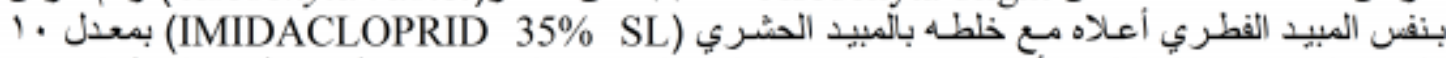

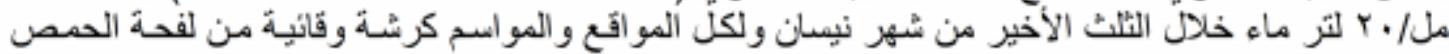

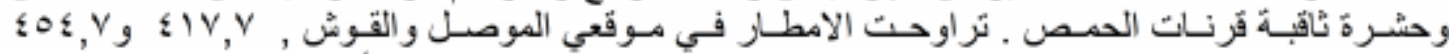

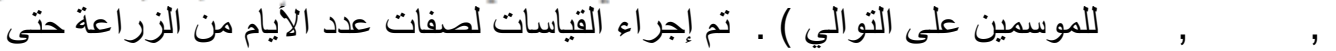

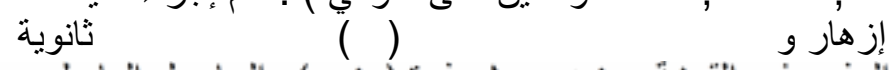

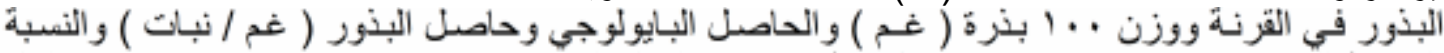

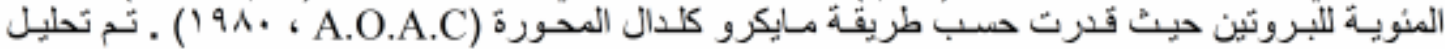

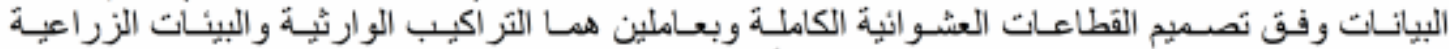

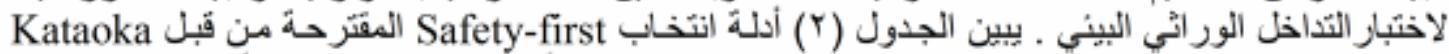

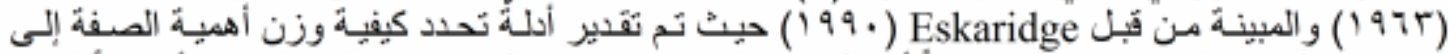

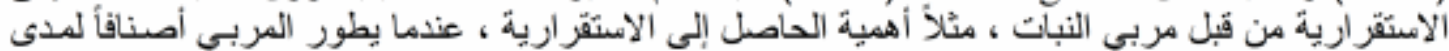

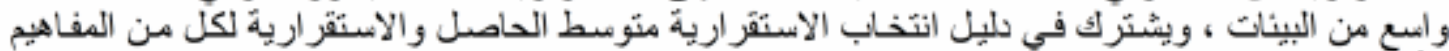

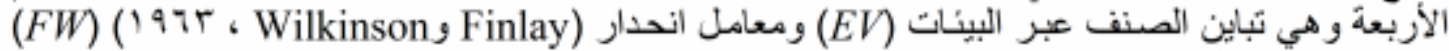

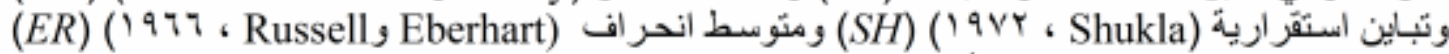

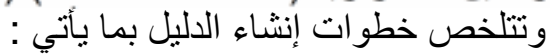

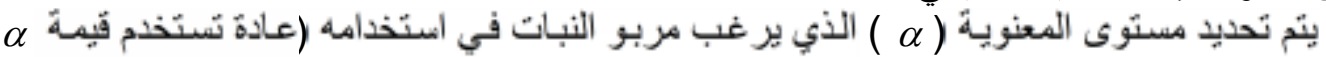

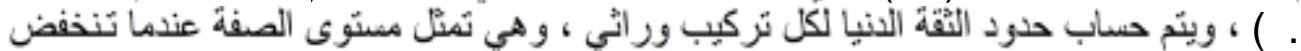

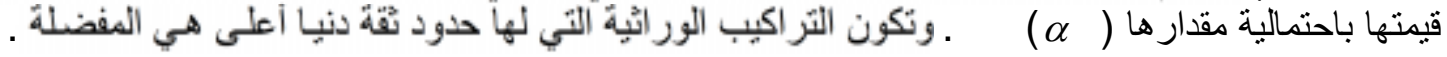



( ) ( )

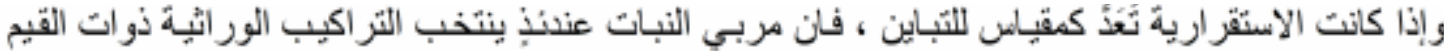

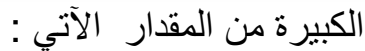

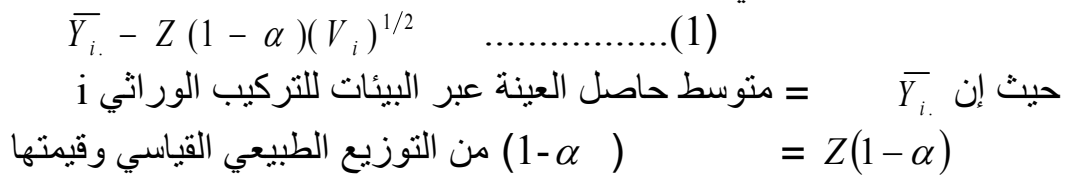
i مقياس لاستقر ارية التركيب الور اثني $=\quad V_{i}$

\begin{tabular}{|c|c|c|}
\hline شكل الدليل للتركيب الوراثي i & & مفاهيم الاستقرارية \\
\hline$\overline{Y_{i .}}-Z(1-\alpha) S_{i}$ & $\mathrm{EV}$ & ـ ـ التباين عبر البيئات \\
\hline $\bar{Y}_{i .}-Z(1-\alpha)\left[(b i-1)^{2} S_{y}^{2}(1-1 / q)\right]^{1 / 2}$ & $\mathrm{FW}$ & Wilkinson Finlay \\
\hline$\overline{Y_{i .}}-Z(1-\alpha)\left[\hat{\sigma}_{E}^{2}+\hat{\sigma}_{i}^{2}\right]^{1 / 2}$ & $\mathrm{SH}$ & - تباين أستقرارية Shukla \\
\hline$\overline{Y_{i .}}-Z(1-\alpha)\left[(b i-1)^{2} S_{y}^{2}(1-1 / q)+S_{b i}^{2}\right]^{1 / 2}$ & ER & 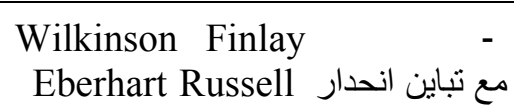 \\
\hline
\end{tabular}

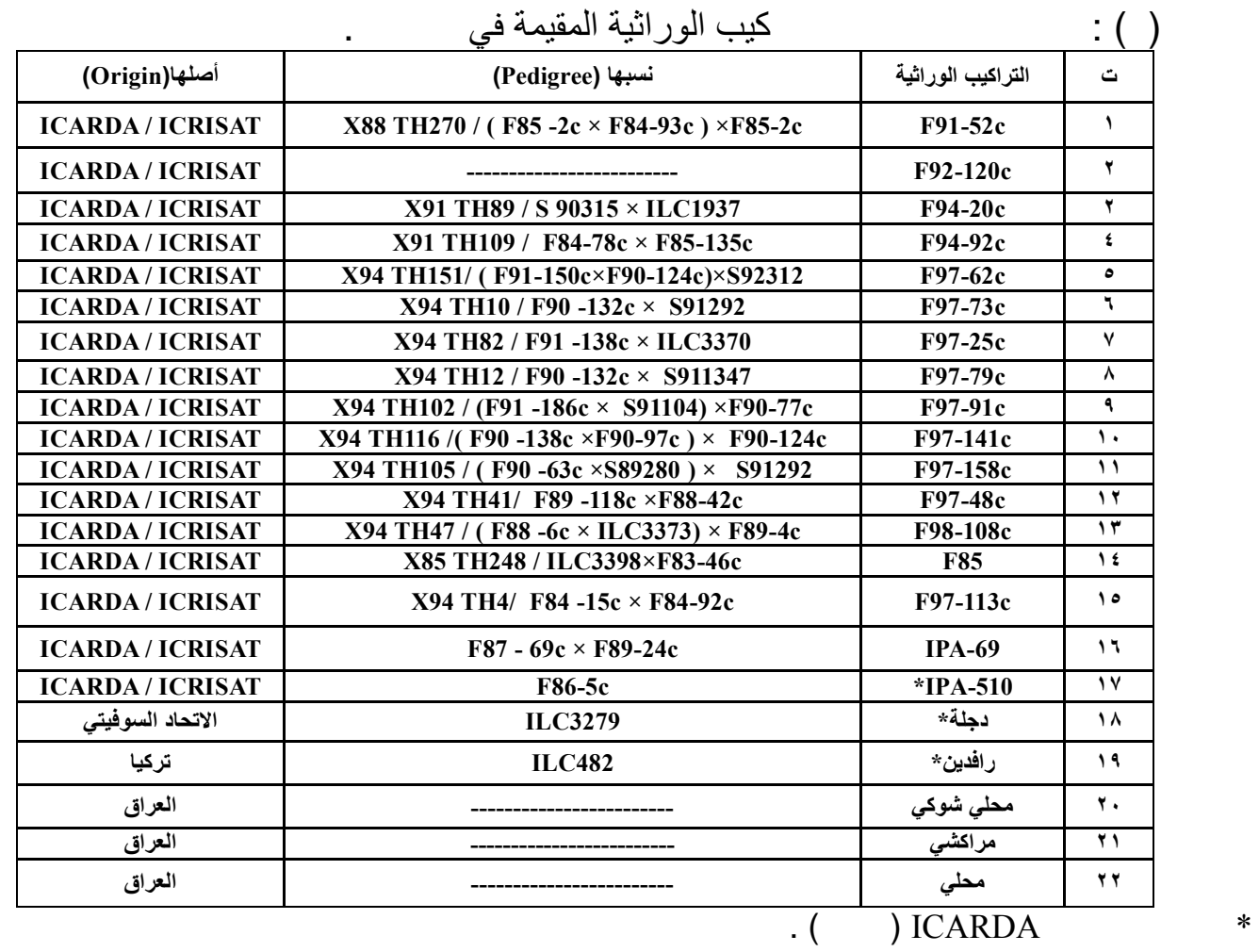

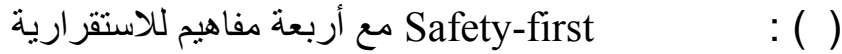

$$
\begin{aligned}
& \bar{Y}_{i .}=\frac{\sum_{j=1}^{q} Y_{i j}}{q} \quad ; \quad \bar{Y}_{. j}=\frac{\sum_{i=1}^{p} Y_{i j}}{p} ; \quad \bar{Y} . .=\frac{\sum_{i=1}^{P} \sum_{j=1}^{q} Y_{i j}}{p q}:{ }^{\prime} \\
& \text { يمثل Yij = حاصل التركيب الور اثي i في البيئة } \\
& \text { p........ = = }=i \\
& =\bar{Y} . . \quad \text {; من البات }=\overline{Y_{i}} \text {. }
\end{aligned}
$$




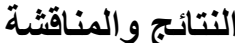

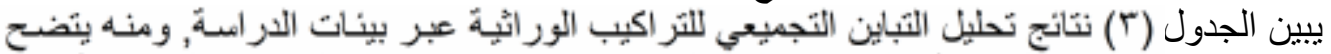

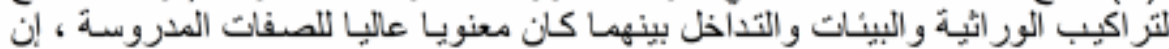

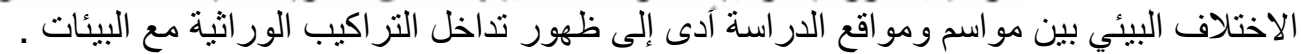

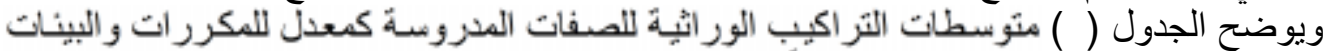

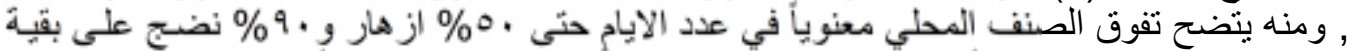
بو مأو وبالترتيب للصفيتي

التراكيب الور اثية تحت الدر اسة إذ أستغرقت التهر

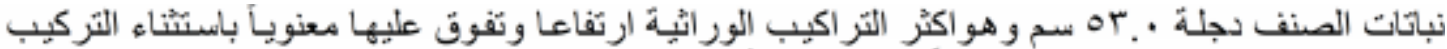

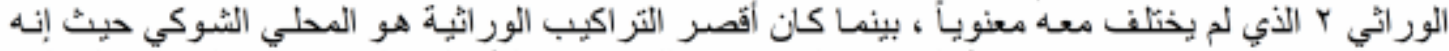

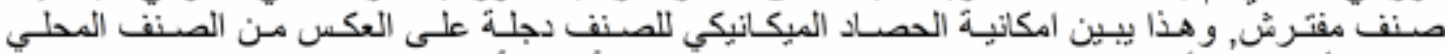

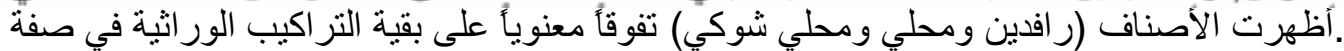

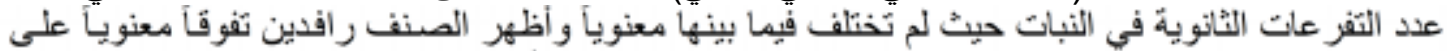

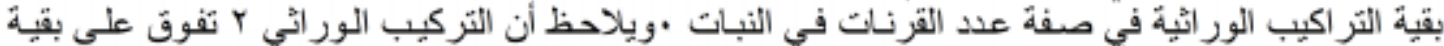

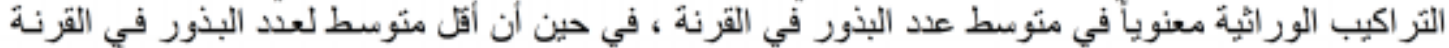

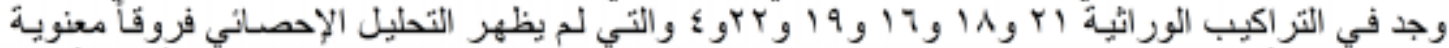

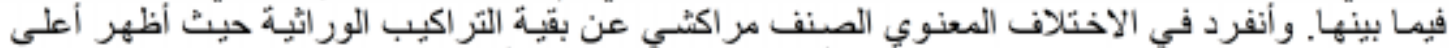

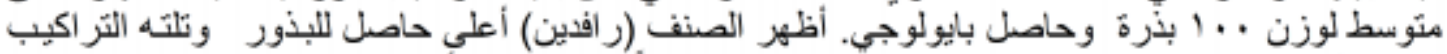

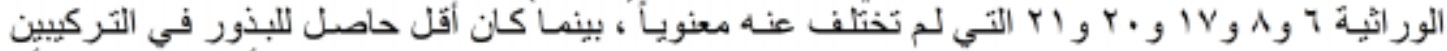

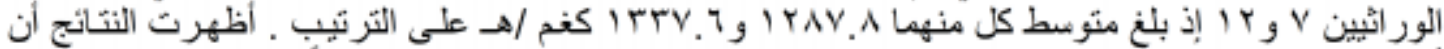

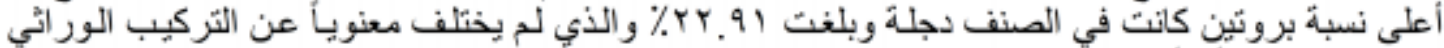

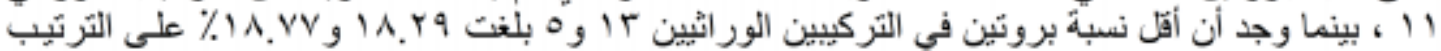

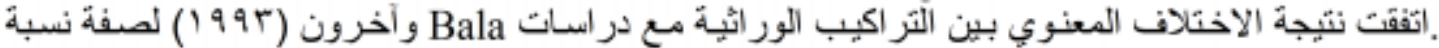

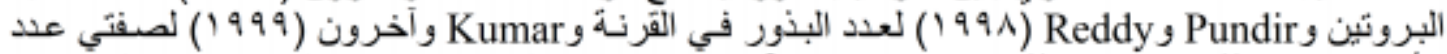

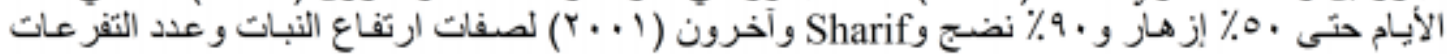

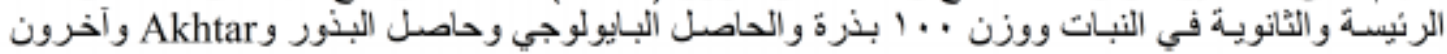

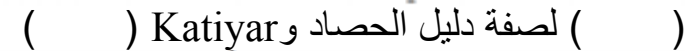

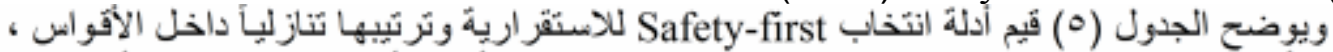

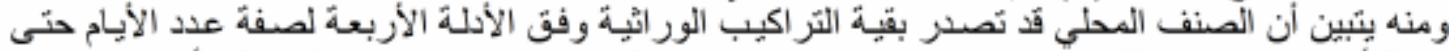

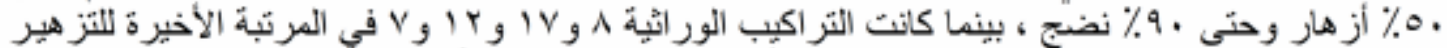

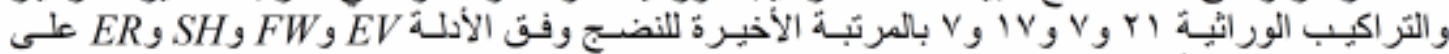

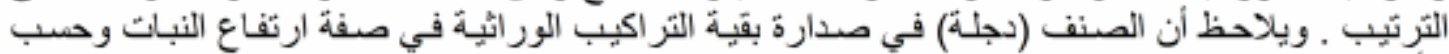
الأدلة

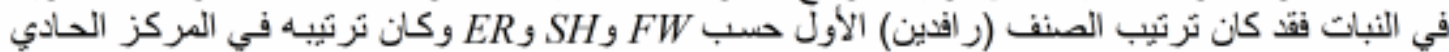

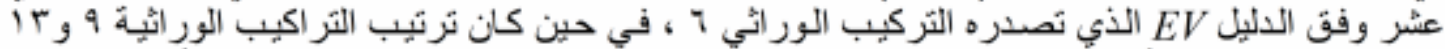

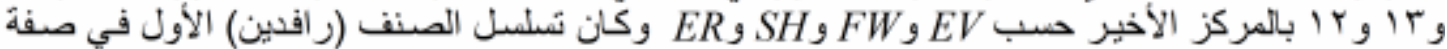

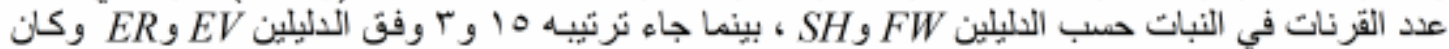

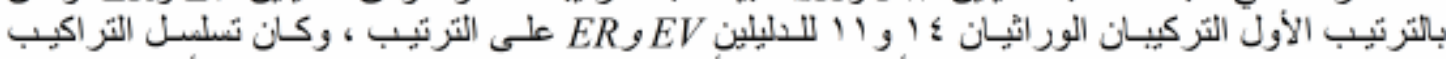

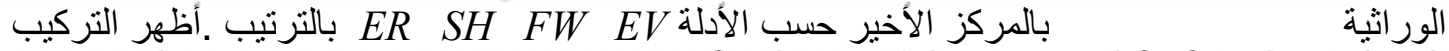
الوراثي 10 المرئبة الأولى في عدد البذور في القَرنة حسب

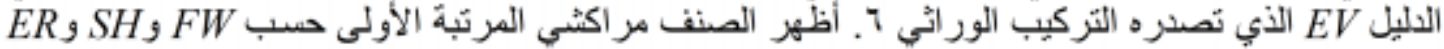

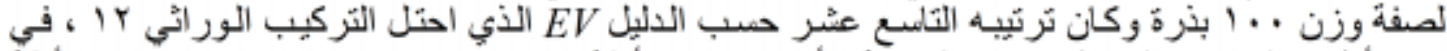

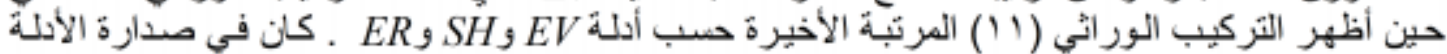

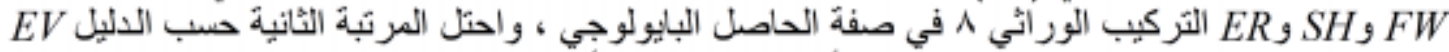

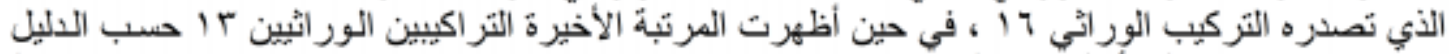

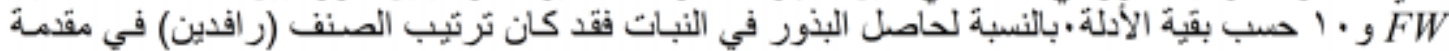

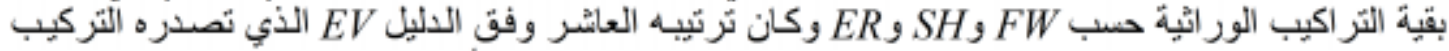

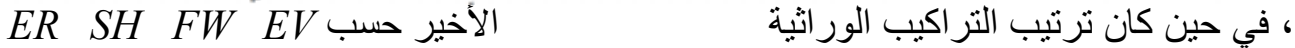

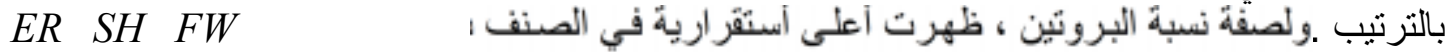




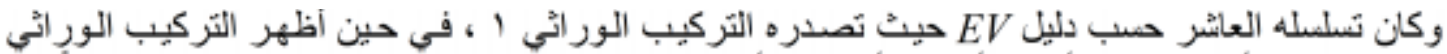

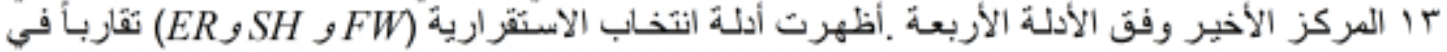

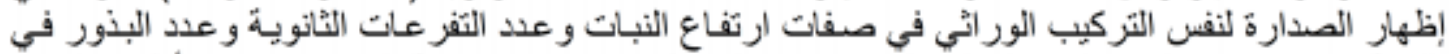

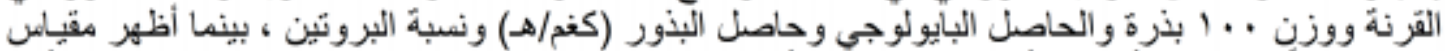

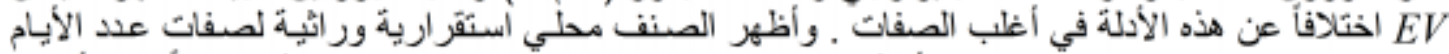

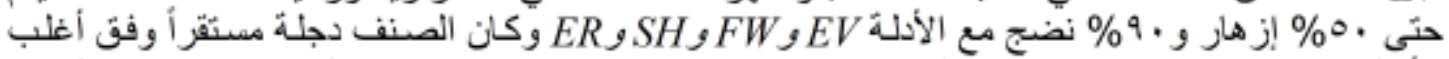

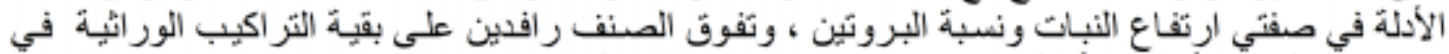

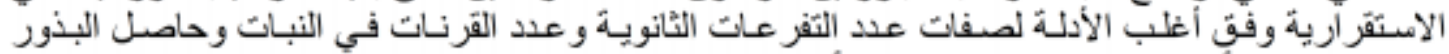

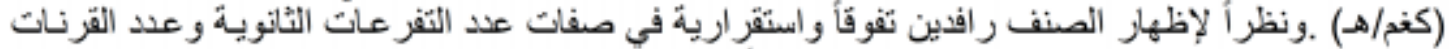

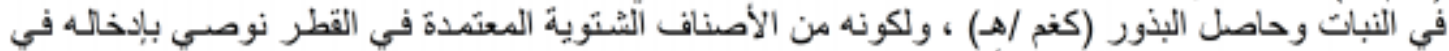

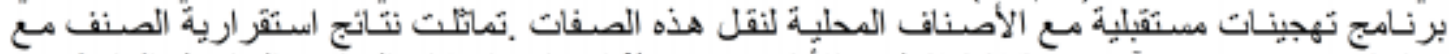

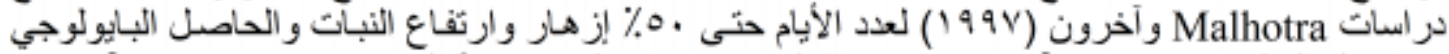

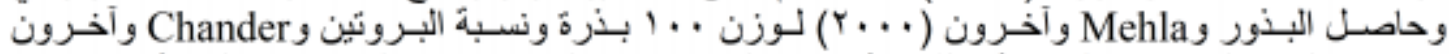

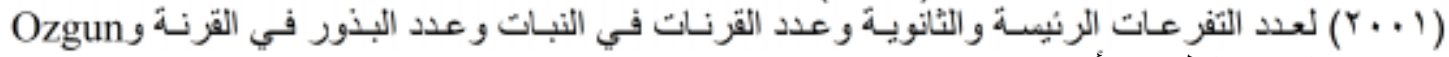

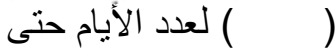

\begin{tabular}{|c|c|c|c|c|c|c|}
\hline & & . & بية للصفات الل & عي للاستقر ار & تلباين الت & ( ): تحليل \\
\hline \multicolumn{5}{|c|}{ M.S } & \multirow[b]{2}{*}{ الحرية } & \multirow[b]{2}{*}{$\begin{array}{l}\text { S.O.V. } \\
\text { S.Oتادر التباين }\end{array}$} \\
\hline & التفرعات الثانوية & () & عدد الأيام & عدد الأيام & & \\
\hline . & . &. & . & . & & \\
\hline$* *$ & $* *$ & $* *$ & $* *$ & $* *$ & & التر اكيب الور اثية \\
\hline$* *$ & $* *$ & $* *$ & $* *$ & $* *$ & & البيئات \\
\hline$* * \quad$. & $* *$ & $* *$ & $* *$ & $* *$. & & التر اكيب الور اثية × \\
\hline . & . & . & . & . & & الخطأ التجريبي \\
\hline \multicolumn{5}{|c|}{ M.S } & \multirow[b]{2}{*}{ الحرية } & \multirow[b]{2}{*}{$\begin{array}{l}\text { Sصادر التباين } \\
\text { S.O.V. }\end{array}$} \\
\hline $\begin{array}{c}\text { البروتين } \\
\text { ( ) }\end{array}$ & () & البايولوجي في ( ) & () & & & \\
\hline$\cdot$ & $\cdot$ & & . &. & & \\
\hline$* *$. & $* *$ & $* *$ & $* *$ & $* *$ & & التر اكيب الور اثية \\
\hline$* *$ & $* *$ & $* *$ & $* *$ & $* *$ & & البيئات \\
\hline$* *$ & $* *$ & $* *$ & $* *$ & $* *$ & & التر اكيب الور اثية"x \\
\hline . & . & & & & & الخطأ التجريبي \\
\hline
\end{tabular}


التر اكيب الور اثية للصفات المدروسة كمعدل للبيئات الخمس .

\begin{tabular}{|c|c|c|c|c|c|c|c|c|c|c|}
\hline نسبة البروتين & حاصل البذور & الحاصل البايولوجي & وزن . . بذرة & عدد البذور في & عدد القرنات في & الثانوية في التفرعات & $\begin{array}{c}\text { ارتفاع النبات } \\
\text { (سم) }\end{array}$ & 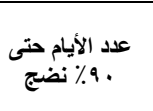 & حتى إزهار & لتراكيب الوراثية \\
\hline $2 r 1 . * A$ & r.r. & $b-\Delta r V \leq q$. & ^.•·r طي & $\Delta \Delta^{\prime} . \cdot V \wedge$ & מ. & ي & $\rightarrow \leqslant \cdot .1$ & $2 j 1+.4$ & ه9 4 ه & $(F 91-52 c)^{\prime}$ \\
\hline r ا طـ ك & و و و & هـ rq01.. & 1. r. r ك ك & $11 . r 91$ & g- د ro.r & צוד. ي ك & Ior.v & Vـ آلاب- هـ & $2 j 91.0$ & $(\text { F92-120c })^{r}$ \\
\hline r Y & و و.ج\& & 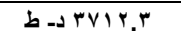 & . & 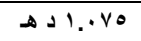 & P- د Y Y P & $b-j \cdot . \cdot v$ & P צ.V & r. r. & b^9.1 & $\left(\right.$ F94-20c) ${ }^{r}$ \\
\hline 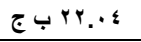 & 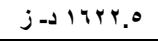 & 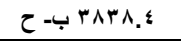 & Jra." & $b$ ح 1.11 & $e^{Y \wedge .0}$ & $j-0 . \mu 1$ & $د \leq Y .1$ & 1.ا ז1 ب- د & ج 9ะ.\& & $(F 94-92 c) \leqslant$ \\
\hline$J 1 \wedge . V V$ & 1.100119 وز & $b \rightarrow r 49.1$ & J S Y Y.० & 1.100 & $9-1$ ro. 0 & $\tau-90.1 \mathrm{~V}$ & r. & j- & زو.. & $\left(\right.$ F97-62c) $^{\circ}$ \\
\hline \& \&.Y & ج & 9 . 9 ـ أ ب & 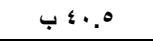 & & J & $j-0.00$ & 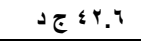 & g & ج 9. & $(\text { F97-73c })^{\top}$ \\
\hline S -ح & 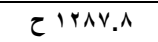 & bحror..l & Zjrr.1 & 1.171 & zjYr.r & & $\Delta \leq \cdot, r$ & r.r.r. & r. & $(\text { F97-25c) })^{V}$ \\
\hline bـ & 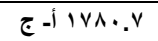 & ج & د & ه & $\Delta \perp$ IY. & $j-د 0 . r v$ & 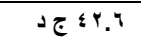 & r.rזו ب ج & 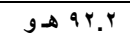 & $(\text { F97-79c })^{\wedge}$ \\
\hline g- د. & 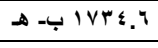 & 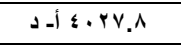 & 244.9 & $g-11 . v \cdot$ & $9-1 r_{0} .0$ & 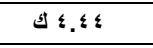 & ". & 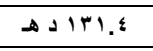 & ד & (F97-91c) $^{9}$ \\
\hline و و و د.A.A. & j1011.. & $b r \leqslant \wedge r$. & Zjry.\& & $g-11 . \cdot V r$ & 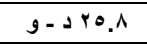 & J & jrצ.V & $j-j|r|$. & $291 . r$ & (F97-141)!. \\
\hline VYrVY أب & r. & b-gr.r.r & r.r. & (1.1.1 & & $b-j \cdot 0$ & jgrv. & bح r.r.r & $b \wedge 9.0$ & $($ F97-158c) \\
\hline S $\tau^{\prime 9.7 V}$ & $\tau^{\prime r r v .7}$ & b $r \leq 4 \leq .7$ & $\Delta r \leqslant .0$ & $\Delta+1 . \cdot \vee \wedge$ & $2+1.7$ & צـ & 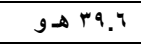 & V. اיا ب- هـ & I94.r & $($ F97-48c) \\
\hline$J 11 . r 9$ & $j 1 \leq 9 \leq .0$ & b-j ro५^.q & 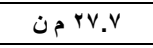 & J $1 . .0 r$ & دY.r & 0.vo ب. ب ج & $\tau^{r \leqslant .1}$ & 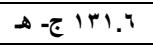 & 19.^و & $\left(\right.$ F98-108c) ${ }^{\prime}$ \\
\hline $5-j 19.9 r$ & " & bـ Mro.9 & $b \tau r 1.0$ & $2 j 1 . . \leqslant 1$ & $e^{Y \wedge .0}$ & ب. & ز و و & bוr... & ي^v.0 & 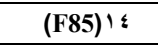 \\
\hline$s-\tau^{\prime 9} . \wedge 4$ & IVץ^.V & 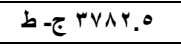 & Ar.r هـ & ؛ & $2 Y Y . \&$ & و & 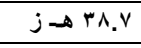 & 9.9.9 (19.9 & زو१.• & $(\text { F97-113c })^{10}$ \\
\hline$\tau-\Delta r \cdot . \leqslant V$ & IVrA.. & ع.1. & r. • ؛ ب ج & b $1 . .1$. & $j-j$ - $r \leqslant .1$ & ه.00 ب.0 - هـ & jor.o & $\tau-g 1 r \cdot . V$ & د9r.A & 14 (أباء997) \\
\hline e r... & 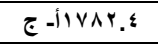 & أ & $e^{r 9 . \varepsilon}$ & ז & . & و - & ك & (1)r.t & I94.r & V|(باء · 101) \\
\hline $\mid$ rr.q1 & ". & 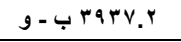 & r. & b) $\ldots v$ & ب9.9 & ל & ior." & j- & ب0.V & ^\ (دجلة) \\
\hline & 119.4 .7 & ز & نrV.r & $b ح 1.10$ & iro.o & 7.7 .1 & $\tau^{r \leqslant . V}$ & J1Yर.० & ي Av.V & 9 (رافدين) \\
\hline 519.84 & ج - أ IV^V.1 & & r.r.r & $g-11 . v \cdot$ & r. & 19.49 & $b r 1.0$ & J S ا I^.V & S 14.7 & • ب (محلي شوكي) \\
\hline$j-د$ r..O. & 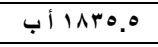 & $f \leqslant r \wedge \wedge . r$ & for.s & b. .99 r & Zjrr.r & و - - - $^{0 . \leqslant V}$ & jrv.r & 1.1 & ي Av.q & ا'ب(مراكثي) \\
\hline $2 r 1 . \cdot 1$ & 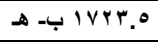 & b-groq9.1 & 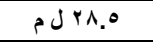 & $b \tau^{1 . .1 V}$ & 1.1.1 ب & 19.4 & $z^{r r .0}$ & P Iro.r & $J \wedge 0.1$ & بr (محلي) \\
\hline
\end{tabular}



( ) ( )
( ISSN $1815-316 \mathrm{X})$
مجلة زر اعة الر افدين

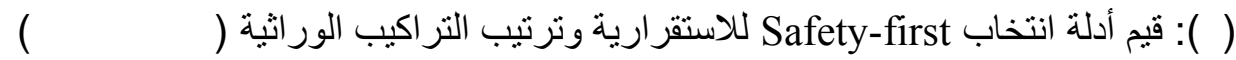

\begin{tabular}{|c|c|c|c|c|c|c|c|c|}
\hline \multicolumn{4}{|c|}{ عدد الأيام حتى ـ 9\% نضج } & \multicolumn{4}{|c|}{ عدد الأيام حتى . • \% ازهار } & \multirow{2}{*}{ التراكيب الوراثية } \\
\hline ER & SH & $\mathbf{F W}$ & EV & ER & SH & FW & EV & \\
\hline$(v)$ (Yv.0 & $(\wedge)^{\wedge \wedge .1}$ & $(\wedge) \mid Y \wedge .1$ & $(\wedge) \mid Y V_{.1}$ & $(1 \cdot)^{\wedge \wedge . \cdot}$ & $(10) \bullet V . \xi$ & $(1 \xi)^{9 \cdot .4}$ & $(14) \wedge A_{.}$. & $($F91-52c) \\
\hline$(r \cdot)^{r r} \cdot r$ & $(1 \wedge)^{\wedge} .^{\top}$ & $(I v) 1 r \cdot .9$ & $(19) 1 \times 9.6$ & 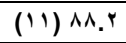 & $(9) \circ 4.8$ & (11) 19.0 & $(I \vee) \wedge \Lambda_{.}{ }^{r}$ & $\left(\right.$ F92-120c) ${ }^{r}$ \\
\hline$(\xi) 1 \times 4.4$ & $(\xi)^{\wedge \uparrow} . \wedge$ & $(r)^{1+4.9}$ & $(\varepsilon) 1 Y 0 . r$ & (V) 14.9 & (?) $0 \leqslant .1$ & (૫) ^v. . & $(\longleftarrow) \wedge r . \wedge$ & (F94-20c) $)^{r}$ \\
\hline$(9)^{1 \times \Lambda_{.} \cdot}$ & $(14)^{\wedge} Q^{-r}$ & (11)1\%9.0 & $(9)$ IrV.V & $(1 \wedge)^{91.4}$ & $(1 \wedge) \circ 9.8$ & (19) 95.7 & $(1 \wedge) \wedge \Lambda .4$ & (F94-92c) \\
\hline$(1 \varepsilon) \times 9.1$ & $(1 ')^{\wedge \wedge} . \wedge$ & $(1+)^{1+r \cdot .}$ & $(1 \wedge)+9.8$ & $(1 \&)^{\wedge 9 .}$ & (11) 04.9 & $(1 \cdot)^{\wedge 9 .} \mathrm{r}$ & $(19) \wedge \wedge . \vee$ & $(\mathrm{F} 97-62 \mathrm{c})^{\circ}$ \\
\hline$(11)^{\prime \prime r \Lambda . Y}$ & $(1 Y)^{\wedge \wedge .9}$ & $(9)^{1+9.4}$ & $(1 \cdot)^{1+\Lambda . \varepsilon}$ & $(10)^{\wedge} \wedge . \Gamma^{\top}$ & $($ (IV) $\bullet 9 . \%$ & $\left(\right.$ (IV) $99^{9} . \xi$ & ('I)Av.r & $\begin{array}{l}\text { (F97-73c) } \\
\end{array}$ \\
\hline$(r+)^{(T) . q}$ & $(r))^{\wedge 9.9}$ & $(Y)^{\prime K r} .1$ & $(I V) \backslash \times q .4$ & $(Y Y)^{9 T . \xi}$ & $(r) \% .1$ & $(r \cdot)^{a r . v}$ & $(I Y)^{\wedge v . \varepsilon}$ & $(\text { F97-25c) })^{v}$ \\
\hline$(I v)^{\prime r} \cdot .1$ & $(r \cdot)^{\wedge} .^{\wedge} \wedge$ & $(17)^{1+\cdot . \wedge}$ & $\left.(1+)^{\prime}\right) \times \Lambda_{. \wedge}$ & $(\text { (IV) })^{\wedge} .4$ & $(1 \varepsilon) \circ V_{. r}$ & $(10)^{91.4}$ & $(r Y)^{\wedge 9.4}$ & $($ F97-79c)^ \\
\hline$(17) \times 9.9$ & $(14)^{\wedge a .} \cdot$ & $(r \cdot)^{\prime r l} \cdot r$ & $(r)+4.9$ & $(\wedge) \wedge \vee . r$ & (I') $\bullet V_{.} r$ & $(\wedge) \wedge \vee .9$ & 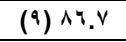 & (F97-91c) 9 \\
\hline$(1+4) 1 \times 9.0$ & $(1 \cdot)^{\wedge \wedge . \top}$ & $(1 \varepsilon)^{1+.} \cdot 0$ & 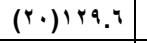 & $(1 Y)^{\wedge \Lambda .4}$ & $(\wedge) \circ 4 . \Upsilon$ & $(11)^{9 \cdot . r}$ & $(r \cdot)^{\wedge \wedge . \vee}$ & (F97-141)'. \\
\hline (T) YrV.Y & 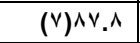 & (?) 1 Tr.q & (ए) & (ฯ) $\wedge$ ^ . & $(v) \bullet \xi . \varepsilon$ & $(v) \wedge v .{ }^{\prime}$ & $(\vee) \wedge \varepsilon . \varepsilon$ & $($ F97-158c) 1 \\
\hline$(10) \times 9.1$ & $(1 \vee)^{\wedge} .^{\circ}$ & $(1+)^{1 r \cdot . r}$ & $(10)+9 .$. & $($ (r) 9 9.9 & $\left.(Y Y)^{T}\right) . r$ & (r) $)^{9 r . \wedge}$ & $(1 \cdot)^{\wedge v .1}$ & $(\text { F97-48c) })^{\prime r}$ \\
\hline$(1 \cdot)^{\prime}+\Lambda^{\prime} .4$ & $(1 \leq)^{\wedge} .1$ & $(19) 1+1.4$ & $(11)^{1+\Lambda .4}$ & (9) ^v.V & $(1 \cdot) \cdot 9.4$ & 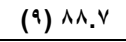 & $(1 \varepsilon)^{\wedge v} . v$ & $(F 98-108 c)^{\top r}$ \\
\hline$(\wedge) \backslash r v . \nu$ & $(T)^{\lambda V .0}$ & $(\mathrm{v}) 1+\Lambda_{.1}$ & (v) IrV.r & $(\xi)^{\wedge} \leq . \Gamma$ & (r) $\circ r_{.} \circ$ & ()$\left.^{\circ}\right)^{\wedge 4.7}$ & (६) $\vee 9 . \xi$ & $(\mathbf{F 8 5})^{1}$ \& \\
\hline$(0) \mid r v .1$ & $(0)^{\wedge V . \varepsilon}$ & $(\xi) I T V^{\prime} T$ & $(0) 1 \times 4.0$ & (14) $\wedge 9.0$ & $(1 Y) 04.9$ & $(1 Y)^{\wedge} \wedge . \vee V$ & $(r) \wedge 9 . Y$ & $(\mathrm{F97-113c})^{10}$ \\
\hline (Ir) & $(9)^{\wedge \Lambda_{.} . r}$ & $(1 \cdot)^{1 \times 9.0}$ & $(17) \times 9.4$ & 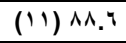 & $(17) \circ \Lambda_{.} \circ$ & $(19)^{91.4}$ & ('I) Av. ${ }^{4}$ & (4 (أباء997) \\
\hline$(r \cdot)^{\prime r} \cdot . q$ & $(Y Y)^{q} \cdot . \varepsilon$ & $($ (r) 1 (r). & $(1 Y)^{1+\Lambda . T}$ & $(r \cdot)^{9 \varphi . V}$ & $(19)^{7 . . V}$ & $(Y Y)^{9} 9.8$ & $(10) \wedge v . \wedge$ & 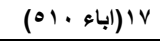 \\
\hline$(1 \wedge)^{1+\cdot .1}$ & $(19)^{\wedge} 9.7$ & $(10) 1+.0$ & $(1\{)+1 \wedge .9$ & $(19)^{91.5}$ & $(r \cdot)^{71.1}$ & $(1 \wedge)^{9 r . \xi}$ & $(\wedge) \wedge 4.0$ & 1 ^ا(دجلة) \\
\hline 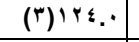 & $\left(\ulcorner)^{\wedge \uparrow .} \cdot\right.$ & $(0) I T V_{. V}$ & (r) Mr.A & $(r) \wedge r . q$ & $(\xi) \circ Y_{.} \circ$ & $(0) \wedge 4.9$ & (ए) V৭.r & (9 (رافين) \\
\hline $\begin{array}{ll}(r)^{\prime r r} . \wedge \\
\end{array}$ & $\left({ }^{(\top}\right)^{\wedge \uparrow .1 \cdot}$ & $(r)^{\prime Y Y . r}$ & $($ (ए) 1 Yr.9 & $(r)^{\wedge r . q}$ & $(\Upsilon) 01.7$ & $($ (५)^ฯ.1 & $($ (५) $\vee 4.9$ & • • ب (محلي شوكي) \\
\hline$(19)^{14} \cdot . r$ & $(10)^{\wedge} .^{4}$ & $(1 \wedge)^{1+1 . r}$ & $(Y Y)^{1+9.9}$ & $(0)^{\wedge 0.1}$ & $(0)$ or. 9 & $(\xi) \wedge \uparrow . \wedge$ & $(0)^{\wedge} \cdot .{ }^{\prime}$ & 1 بر (مركثي) \\
\hline$(1)+r+r$ & $(1)^{\text {Ar. }}$ & (1) 1 1 4.9 & (1) $110 . r$ & (1) $\wedge,{ }^{\wedge}$ & $(1)^{0} .$. & (1) ${ }^{\wedge 0 .} \cdot$ & (1) vr. & بr (محلي) \\
\hline \multicolumn{4}{|c|}{ عدد التفرعات الثانوية في النبات } & \multicolumn{4}{|c|}{ ارتفاع النبات (سم) } & |s \\
\hline ER & SH & FW & EV & ER & SH & FW & EV & المزاريب الهونايكي \\
\hline$(1 \cdot)^{4} .7$ & $(10) \leq . Y Y$ & $(17)^{\varepsilon . \wedge 1}$ & $(I V)^{r} . v \wedge$ & $(9)^{4} \times .9$ & $(1 \cdot)^{\mu 1 . \wedge}$ & $(1 \cdot)^{r 9.8}$ & $(0)^{r v} v^{r}$ & (F91-52c)' \\
\hline$(1 \wedge)^{4.99}$ & $(19)^{r} . A r$ & $(1 \wedge) \leqslant . \leqslant \wedge$ & $(r \cdot)^{r} . \mathrm{IV}$ & $(\ulcorner) \leqslant \Gamma . \wedge$ & $(\Upsilon) \leqslant 1.7$ & $(\Upsilon)^{01.0}$ & $(1 \Lambda)^{r \Lambda . \Lambda}$ & $\left(\right.$ F92-120c) ${ }^{r}$ \\
\hline$(1 T) \leqslant . \leqslant V$ & $(1 \xi) \leqslant . T V$ & $(14)^{5.94}$ & $(1 T) \leqslant .90$ & $(4)^{r a .1}$ & ()$^{r} \div .0$ & $(r) \leqslant \Upsilon . \Upsilon$ & $(r)^{r v . v}$ & $\left(\right.$ F91-52c) ${ }^{r}$ \\
\hline$(0) \leqslant . V 1$ & $(4) \leq .0 Y$ & $(0) 0 . Y \Lambda$ & $(\Psi)^{\xi} . \vee ५$ & $(\wedge)^{\mu q} . \cdot$ & $(\wedge)$ rr.9 & $(7) \leqslant 1 . V$ & $(r)^{r v . q}$ & $($ F94-92c) \\
\hline$(\mathrm{IV}) \leq .1 \mathrm{.1T}$ & $(I V) \leq .4$ & $(I V) \leq . V$. & $(10) \leqslant .1 Y$ & $(\Gamma) \leqslant \cdot .9$ & $(r)^{r 0.9}$ & $(\xi) \leqslant Y .4$ & (4) 4.0 & $(\mathrm{F97-62c})^{\circ}$ \\
\hline$(\xi) \leqslant . V Y$ & $(0) \leqslant .0 \leqslant$ & $(4)^{0} . Y \varepsilon$ & $(1)^{4.199}$ & $(\xi) \leqslant \cdot . \mu$ & $(0)^{r} \leqslant .1$ & $(0) \leqslant \varphi .1$ & $(1)^{\mu \Lambda_{*} \cdot}$ & (F97-73c) \\
\hline$(11) \leq .0 Y$ & 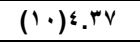 & $(1 \varepsilon)^{\varepsilon .19}$ & $(9) \leqslant . \leqslant 4$ & $(1 \leqslant)^{4} \leqslant .4$ & $(1)^{\mu} \cdot .^{v}$ & $(1 \varepsilon)^{r v} \cdot r$ & $(\wedge)^{r} \varepsilon .9$ & $\left(\right.$ F97-25c) ${ }^{v}$ \\
\hline$(19)^{r .94}$ & $\left({ }^{\prime \wedge}\right)^{r .90}$ & $(\Lambda)^{0.14}$ & $(19) \leq .11$ & $(v)^{r 9}$. & $(v)^{r} \xi \varphi^{r}$ & $(v) \leq 1 . v$ & $(\xi)^{r v . \varepsilon}$ & $($ F97-79c)^ \\
\hline$(r \cdot)^{r . v 4}$ & $(r \cdot)^{r .09}$ & $(r \cdot)^{4} . r^{\prime}$ & $(r+)^{r .0 v}$ & $(1 \cdot)^{4}+.{ }^{\prime}$ & (9)rr.r & $(\wedge) \leqslant 1 . \Gamma$ & $(v)^{r}=.4$ & $($ F97-91c) \\
\hline$(1 \leq) \leq . \leqslant \leqslant$ & $(11) \leq . \% 0$ & $(V)^{0} . r \mu$ & $(v) \leqslant .0 \%$ & (10)rr.4 & $(14)^{r \Lambda . r}$ & $(r \cdot)^{r} \varepsilon . r$ & $(I v)^{r a . r}$ & (F97-141)'. \\
\hline$(?) \leq . \vee \cdot$ & (9) $4 ., 49$ & $(1 \cdot)^{4.91}$ & $(1 \cdot) \leqslant . T T$ & $(1 \Gamma)^{\mu} \leqslant . V$ & $(1 \varepsilon)^{Y 9 . \varepsilon}$ & $(1+)^{r v . \varepsilon}$ & $(1+r)^{m} \cdot A$ & $($ F97-158c) 1 \\
\hline$\left(Y^{\prime}\right)^{\Gamma .0 \xi}$ & $(r))^{r} \leq \leqslant$ & $($ (Y) $\{.11$ & $(r) r \cdot \cdot \wedge$ & $(14)^{r Y . \&}$ & $(10)^{\times 9.4}$ & $(10)^{m} \times .1$ & $\left(I^{r}\right)^{r Y .9}$ & $(\text { F97-48c) })^{\prime Y}$ \\
\hline$(r))^{r} . r \Lambda$ & $(r Y)^{r}$ r.\&r & $\left(r^{Y}\right)^{r} .9 \xi$ & $(19)^{4.54}$ & $(1 \wedge)^{r 1.1}$ & $(19)^{4} \times .8$ & $(1 \wedge)^{\mu} \leqslant . v$ & $(19)^{r} \leqslant$. & (F98-108c) \\
\hline$(\wedge) \leqslant .70$ & $(\Psi) \leq .4 \pi$ & (घ) 0.71 & (7) 4.04 & (1') ro.v & $(1+)^{\mu} \cdot . \cdot$ & $(1)^{r v . v}$ & (1'1)rr.r & $(F 85)^{1 \leqslant}$ \\
\hline$(r) \leq . \vee \wedge$ & $(\xi) \leqslant .00$ & (11) $\varepsilon .9 v$ & $(r)^{\varepsilon} . \mathrm{YV}$ & $(1 Y)^{r 0.4}$ & $(1 Y)^{\mu} \cdot . \varepsilon$ & $(1 r)^{r v} .0$ & $(9)^{r} \leqslant .0$ & $(\text { F97-113c) })^{10}$ \\
\hline$(9) \leq .7 Y$ & $(\wedge) \leqslant . \leqslant \varepsilon$ & $(1 r) \leqslant .9 r$ & $(0) \leq .7$ & $(19)^{r} \cdot . v$ & $(1 \wedge)^{r v} . v$ & $(14)^{4} 4.8$ & $(17)^{49.7}$ & (4) (أباء99) \\
\hline$(v) \leq . v \cdot$ & $(v) \leqslant .01$ & $(9)^{0} .$. & $(\xi)^{\xi} . V Y$ & $(0) \leqslant \cdot$ & $(\xi)^{10.9}$ & $(9) \leqslant \cdot .4$ & $(1 \cdot)^{\mu \varepsilon} .^{\mu}$ & 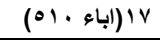 \\
\hline$(1 Y) \leqslant .01$ & $(1 \Gamma)^{ \pm . Y \Lambda}$ & $(10) \leqslant . \wedge \wedge$ & $(1 \xi) \leqslant . Y Y$ & $(1) \leqslant v_{.} r$ & $(1) \leqslant r . \varepsilon$ & (1)०r.. & $(1 \xi)^{r a .1}$ & 11 (دجلة) \\
\hline$(1)^{0 . \wedge r}$ & $(1)^{0.19}$ & $(1)^{4} .89$ & $(1) \leqslant . \Gamma$ & $(r) r)^{r .9}$ & $(r)^{r} \varepsilon . r$ & $(19)^{\mu} \leqslant . r$ & $(r \cdot)^{r} \cdot r$ & 99 \\
\hline$(r)^{0.7}$ & $(r)^{0 .} \varepsilon^{\top}$ & ()$\left.^{4}\right)^{4} . \cdot \cdot$ & $(\wedge) \leq . \leqslant V$ & $(r Y)^{r 0.9}$ & 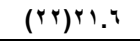 & $($ YYYY.Y & $(Y Y)^{\prime} \leqslant .1$ & · ب (محلي شوكي) \\
\hline$(10) \leqslant .+1$ & $(17) \leq .1 Y$ & $(19) \leqslant . \leqslant 0$ & $(T Y)^{\leq} . Y \wedge$ & $(I v)^{r}+. v$ & $(I v)^{r v . q}$ & $\left({ }^{\prime} v\right)^{\mu} \varepsilon . v$ & $(10)^{49.1}$ & 1 (مراكثي) \\
\hline$(17)^{5.19}$ & $(1 Y) \leqslant . \% 0$ & $(5)^{4.14}$ & $(1 \wedge)^{r} . v_{0}$ & $\left(r^{r} \cdot\right)^{r q . r}$ & $(r \cdot)^{r \xi . \wedge}$ & $(r+)^{r+1}$ & $(r) r \cdot)^{r}$ & r r (محلي) \\
\hline
\end{tabular}




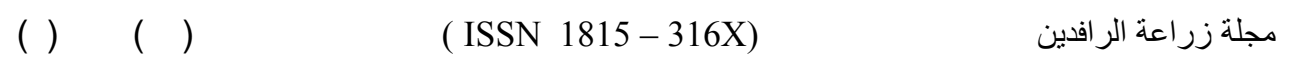

( )

\begin{tabular}{|c|c|c|c|c|c|c|c|c|}
\hline \multicolumn{4}{|c|}{ عدد البذور في القرنة } & \multicolumn{4}{|c|}{ عدد القرنات في النبات } & \multirow{2}{*}{ التراكيب الوراثية } \\
\hline ER & SH & FW & EV & ER & SH & FW & EV & \\
\hline$(1 \cdot) \cdot .91$ & ) $.9 \mathrm{~V}$ & $1 . .0$ & (1) .99 & $4)^{r} .94$ & $(9) 10.0 Y$ & $1)^{r 4 . .4}$ & 7) & $(\mathrm{F91-52c})^{\prime}$ \\
\hline$(10) \cdot .94$ & ) $.9 \mathrm{v}$ & 1.15 & $(Y Y) \cdot . \vee \wedge$ & YY.IV & 1.) 1 \leqslant .94$ & )$^{r \leqslant . \leqslant 1}$ & ()$^{r}, . r v$ & $\overline{(F 92-120 c)^{r}}$ \\
\hline (?) $1 . .1$ & .99 & 1.9 & ) $1 . .4$ & $r \cdot .00$ & r) 18.99 & r) & $4)^{r} \cdot .4$ & \\
\hline$(15) \cdot .9 \mathrm{~V}$ & o) $.9 \xi$ & 7) $1 . .1$ & 7). .9. & Pr.l. & $1919 . \mathrm{V}^{2}$ & 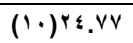 & $(0)^{r} 1.71$ & $\overline{(F 94-92 c) \leqslant}$ \\
\hline$(\Upsilon) ! . \cdot v$ & 11.0 & $1 . .9$ & $(\wedge) \cdot .99$ & )$! \leqslant . \wedge \wedge$ & A) 11.01 & $\cdot)^{\Gamma ! . \top \Lambda}$ & $1 \wedge) 10.01$ & $\left(\mathrm{F97-62c}^{\circ}\right.$ \\
\hline$(\Psi) 1 . \cdot \Gamma$ &. .1 & $1 . .1$ & $1 . .4$ & $\mid)^{r \cdot . r}$ & o) & e)rr.9v & 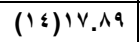 & $($ F97-73c) \\
\hline$(\wedge) \cdot .99$ & $1 \ldots$ & 1.1. & $(1 T) \cdot .9 \xi$ & 10.9 & 1) $1 . .01$ & TY.YA & .011 .99 & \\
\hline$(\xi) ! \cdot r$ & (v) 9.99 & $(1+1) 1 . r$ & $1 . .1$ & Rr... & $10 . v 1$ & rะ.91 & (Y)YY.94 & \\
\hline$(1 \leq) \cdot .9 \mathrm{~V}$ & $(1 \xi) \cdot .99$ & (9) $1 . \cdot 4$ & ) .91 & $r \cdot . \quad v \leqslant$ & r) $1 \varepsilon .0 v$ & 4) & $1 \cdot)^{r} \cdot . \leqslant 0$ & c) $)^{9}$ \\
\hline (1). .91 & $(9) \cdot .9 \mathrm{~V}$ & $\left.(1 \cdot)^{\prime}\right) . .4$ & $(\mathrm{v}) \cdot .99$ & Y) $1\{.19$ & 9) $11 . \mathrm{rr}$ & 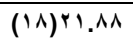 & 9)10.rr & (F97-141) \\
\hline$(9) \cdot .99$ & (11)..9v & $(1 \xi) 1 . r$ & (11) $\cdot .9 v$ & 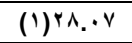 & $r)^{r ! . r \wedge}$ & $r)^{r \cdot . \vee \wedge}$ & $(\xi) Y T^{\prime} \cdot r$ & $($ F97-158c)"। \\
\hline$(0) 1 . \cdot r$ & ) .99 & $(11) 1 . .4$ & $(r) 1 . \cdot r$ & A) & $(r \cdot) 1 . .99$ & r) r...o & 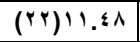 & $(\text { F97-48c })^{\prime r}$ \\
\hline$(1 Y) \cdot .91$ & $(15) \cdot .99$ & $(\wedge) 1 . \cdot 0$ & $(1 \cdot) \cdot .9 v$ & 19.91 & $(1) 1$ (1).vo & 14) & $(11)^{r} \cdot \varepsilon 1$ & $(F 98-108 c)^{\prime r}$ \\
\hline (v) $1 .$. & $(I Y) \cdot .9 \mathrm{~V}$ & $(1, Y) \cdot . r$ & r). .94 & T\&..r & (?) 1 Y.va & 1) & (1) & $(F 85)^{1 !}$ \\
\hline (1)1.11 & 1.9 & 11.10 & (o) 1... & 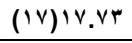 & $(1 \xi) T . \wedge r$ & TE.VA & $(1 \Gamma)^{\prime \wedge . \vee \wedge}$ & (F97-11 \\
\hline$(r Y) \cdot . \wedge \tau$ & $(Y Y)$. & $(r) \cdot .94$ & $(r \cdot) \cdot . \wedge r$ & & & זי. & 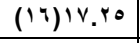 & \\
\hline$\left(r^{\prime}\right) \cdot .91$ & & & & & & 1.91 & $(1 \mathrm{~V}) 19.0 \mathrm{~V}$ & 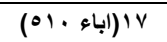 \\
\hline$(1 \wedge) \cdot .9 \leqslant$ & & & & & & & $(1 Y)^{19.4 r}$ & 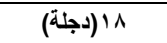 \\
\hline$(\mathrm{IV}) \cdot .90$ & ('v) & $(19) . .9 v$ & (1) & ro.v4 & 10 & 4 & $(10)^{\prime Y} \mathrm{Y} . \mathrm{TV}$ & \\
\hline (19)..9r & $(1 \wedge)$. & $(1$ & $(1$ & & & & & 5 \\
\hline$(r \cdot) \cdot .9 r$ & (r) $\left.r^{\prime}\right) \cdot 9$. & $\left(Y^{Y}\right) \cdot$ & $(r) \cdot . \wedge r$ & .91 & $(Y Y)^{\prime}{ }^{\prime} \cdot{ }^{\prime} \cdot$ & $(r Y)^{19.10}$ & $\left(r^{\prime}\right)(1, .7 \varepsilon$ & اب(مر اكثم) \\
\hline & 9.94 & & & & $(r)^{r} \cdot . \cdot 1$ & ro & & \\
\hline \multicolumn{4}{|c|}{ الحاصل البايولوجي في النبات(كفغ/هـ) } & \multicolumn{4}{|c|}{ وزن .. ب بذرة } & \multirow{2}{*}{ التراكيب الوراثيّة } \\
\hline & $\mathrm{Gr}$ & & $\mathrm{v}$ & & SH & $F W$ & $\mathbf{E V}$ & \\
\hline$(19)^{\text {rVYT.Y }}$ & $(14) 1 \wedge+9.9$ &.$r$ & $\xi)^{\Upsilon \wedge \wedge 0 . \eta}$ & $(1 \varepsilon)^{r V . T \xi}$ & (IT) rY.TV & $9 . r 4$ & T) Yo. $2 \mathrm{~A}$ & ) \\
\hline (Y) YYAV. 4 & & & & & & A. & r. & (F92 \\
\hline$(\wedge)$ rrvv. 9 & $(1$ & & & & & & & \\
\hline$(1 \%)^{r \cdot 10 . r}$ & $(1 \cdot)^{r} \cdot \Lambda_{. \varepsilon}$ & & & & & & & \\
\hline 101.4 & & & $(\mathrm{lin})$ & & & 1.94 & & ec) $)^{0}$ \\
\hline 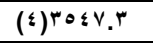 & & 9 & & & & & .74 & \\
\hline 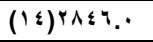 & 494.5 & 1.9 .1 & $v 1.1$ &.$v \varepsilon$ & $r .91$ & $9 \xi$ & $.0 \xi$ & \\
\hline (1) rv99.v & 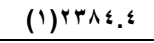 & va.9 & 94.9 & &.$\wedge \mu$ & $0 .{ }_{0}{ }^{2}$ &.$v_{0}$ & c)^ \\
\hline (0) $4 \leqslant 04, \wedge$ & & & $v^{2}$ & & & & T.r4 & \\
\hline $9 \cdot r^{r}$ & $r A ! . r$ & $\leqslant \wedge$ & .8 & & $\cdots$ & 9.54 & .89 & $(\mathrm{~F} 9$ \\
\hline$\cdot \cdot \cdot \cdot .9$ & $\leqslant \varepsilon \cdot .4$ & & 9.9 & & .99 & 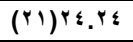 & r.Ar & (F9) \\
\hline VVAo.. & ri.. & $\theta \cdot . r$ & o^v. 9 & & .00 &.$v \varepsilon$ & $1 . \cdots$ & \\
\hline$(10)^{\text {YATQ. }}$ & 119.9 & $. T \leq . V$ & vir.o & 8.9 & $9 . q^{9}$ & $(r \cdot)^{r \xi \Delta . A r}$ & $(1 \wedge)^{19 . \wedge 9}$ & $(F 98-108 c)^{1 T}$ \\
\hline (9)retv. & $19 \cdot v . v$ & $r \cdot 1.9$ & $(1 \cdot)^{\text {riv? }}$ & r9.9\% & (Ir)rT.AT & (11) r..vo & $(v)^{r \Lambda .} \cdot 9$ & $(\mathbf{F 8 5})^{1}$ \\
\hline$(1 \wedge)^{\text {YVT }}$ & $(1 \leqslant) 1)^{\prime} 0 \Lambda_{.} 0$ & $($ (Ir) rion.1 & $(1 T)^{\text {YA9 }}$ & $r \cdot .99$ & $(\Lambda)^{r 0 .{ }^{\prime} \Lambda}$ & $(1 \cdot)^{r} \cdot . \wedge \wedge$ & $(r)^{r a . \wedge 0}$ & $($ F97-113c) 10 \\
\hline$(r)^{r+111 . r}$ & $(0)^{r} / \wedge 0.1$ & $(\Gamma)^{\Upsilon \wedge a ५ . \wedge}$ & $(1)^{r 4.4 .1}$ & (ץ) ฯฯ.४. & $($ (r) ry... & $(r)^{r \lambda .4 r}$ & $(\wedge)^{\Upsilon ฯ} . \wedge \wedge$ & (17 (أباء99) \\
\hline$(r)^{r 09 . .0}$ & rros.9 & (V)rvav.r & (v) rere.9 & 100.10 & $(\xi)+.4 \pi$ & )$^{r 4 . \wedge \Lambda}$ & $(9)^{r} Y . v V$ & 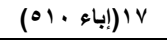 \\
\hline$(r \cdot)^{r}+\Lambda, . r$ & $(1 Y)^{194 V .9}$ & $(?)^{M \wedge \mu \Sigma}$ & $(10)^{r \Lambda .9 .0}$ & (ri)rerrer & $(r))^{\text {IN.rV }}$ & $(1 M)^{Y Y . Y \&}$ & (r) (r)T.ru & 1^ (دجلة) \\
\hline$(1 \cdot)^{r+00.1}$ & $(9)^{4}+74.9$ & $(14)^{\mu} \div 9 \Lambda . V$ & $(9)$ rema.1 & $(r \cdot)^{r+. \& r}$ & $(r \cdot)^{11} .09$ & 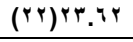 & $(r \cdot)^{10.7 r}$ & 9 \\
\hline १६ &.$\wedge$ & & & ' & Y & 90 & $\leqslant$ & شبو (محلمي \\
\hline 1००.० & & & & & & 10 & & 1 براكثي \\
\hline$(17)^{\times 117.9}$ & (IV) IVar. & $(1 \wedge)^{r} \leqslant 04.0$ & $(14)^{\Upsilon \Lambda} \cdot{ }^{\prime} \cdot{ }^{\top}$ & $(1 v)^{r e . v_{0}}$ & $(1 \Lambda)^{r} \cdot . r T$ & $(19)^{Y 0.10}$ & $(I V)^{19 . r Y}$ & rr (محلي) \\
\hline
\end{tabular}




\begin{tabular}{|c|c|c|c|c|c|c|c|c|}
\hline \multicolumn{4}{|c|}{ نسبة البروتين (٪) } & \multicolumn{4}{|c|}{ حاصل البذور في النبات(كغ/هـ) } & \multirow{2}{*}{ التراكيب الوراثية } \\
\hline ER & SH & FW & EV & ER & SH & FW & EV & \\
\hline$(0)^{r} \cdot . \cdot A$ & (ग) 19.11 & (7) $r . .91$ & (1) $19 . \wedge r$ & $(19)^{1} 1 . \leqslant r . r$ & $(I V)^{\wedge} \leqslant . .0$ & .019 .4 .0 & $($ (IV) 1119.1 & $($ F91-52c) 1 \\
\hline$(\wedge)^{19.1 \%}$ & $(9)^{\wedge \wedge . \vee 1}$ & $(1 \%)^{19.4 \pi}$ & $(I Y)^{\wedge \Lambda_{.} Y V}$ & $(v) 1 \leqslant 9 \%, Y$ & $(\wedge) 1.04 . \wedge$ & $(9) 1710 .$. & $(1) 1011.0$ & $\left(\right.$ F92-120c) ${ }^{r}$ \\
\hline$(19)^{\prime \prime 4 . r 4}$ & 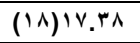 & $(1 \xi)^{19 . \%}$ & $(1 \xi)^{1 Y .01}$ & $(1 \cdot)^{1 \leqslant \uparrow 9.9}$ & $(11) 1.49 .4$ & $(1 Y)^{109.9}$ & $(\wedge) 1 \leqslant 0 \leqslant .4$ & $(\text { F91-52c) })^{r}$ \\
\hline$(4)^{19 . \wedge r}$ & $(0)^{19 . \wedge \varepsilon}$ & $(\xi)^{r 1.04}$ & $(\vee) \backslash \wedge . \vee \varepsilon$ & (IV) $110 \%$. & $(10)^{\wedge \wedge \vee . \varepsilon}$ & $(1 \wedge) 1 \leqslant r 1 . v$ & $(1 \varepsilon) \backslash 1 \wedge \wedge . \varepsilon$ & $\left(\right.$ F94-92c) ${ }^{4}$ \\
\hline (14) & (IV) IV.ra & $(19)^{11.01}$ & $(r))^{10.4 \Lambda}$ & $(r \cdot)^{9 \wedge 4 . \%}$ & $(19) \times \times 4.9$ & $(I V) \geq \leqslant \varepsilon . .7$ & $(19) 1.18 .1$ & $\left(\right.$ F97-62c) ${ }^{\circ}$ \\
\hline$(r)^{r} \cdot . T^{r}$ & $(r)^{r} . .0 \leqslant$ & Yr.V & $(\xi)^{1 \wedge .9 .}$ & $(\Gamma)^{\prime}$ & $(\Upsilon)^{\prime}$ & .9 & 4.5 & (F97. \\
\hline$(I v) \operatorname{lv.\Delta v}$ & $(10) 1 \% .01$ & .17 & $(17)^{\prime V . r A}$ & $(r)$ & $(r)$ & $(r)$ & r) $00 . .0$ & $\left(\mathrm{F97-25c)^{v }}\right.$ \\
\hline$(1 \mathrm{l})^{1 \wedge .1 \mathrm{~V}}$ & $(I Y)^{\wedge A . r}$ & $(1 \cdot)^{r \cdot . \cdot 1}$ & $(11)^{\wedge \Lambda . \& 1}$ & $(7)^{1} 1899 . \wedge$ & $(\xi) 11+9 . \xi$ & $(\Gamma) \operatorname{lv\mu } 0.1$ & $(9) 1 \leqslant 0 . .1$ & $(\mathrm{F97-79c})^{\wedge}$ \\
\hline$(v)^{19.01}$ & $(v) / 9.4 Y$ & $(9)^{r} \cdot . .4$ & $(ץ)^{19.01}$ & $(\xi)^{1019 . \xi}$ & $(4) 11.9 .9$ & $($ (^) $14 \times 9.9$ & $1 \div \div 91 . \wedge$ & $($ F97-91c) 9 \\
\hline$(1 \cdot)^{11 .} .14$ & $(1 \cdot)^{1 \wedge .01}$ & $(11) 19.10$ & 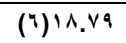 & (YY)VYa. & (YT) TYY.A & (r.)ITIr. & $(r \cdot)^{\wedge} 11.1$ & (F97-141) \\
\hline$(\xi)^{r} \cdot . r q$ & $(\xi)^{r} \cdot . r \mu$ & $(r)^{r}+1 . T$ & $(9) \backslash 1.7 \varepsilon$ & $(\wedge)^{\prime} \leq 49 . \wedge$ & $(9) 1.04 . V$ & $(1 \xi)^{10 Y \varepsilon . \wedge}$ & $(\xi) \leqslant$ IV.Y & (F97-158c)"' \\
\hline$(1 \varepsilon)^{1 \% . v q}$ & $(1 \xi) Y V . V \varepsilon$ & $(10)^{19.11}$ & $(1 A)^{\prime 18 . r a}$ & $(1 \varepsilon)^{\prime 1 \vee v \vee .1}$ & $(r \cdot)^{r+4 .} \cdot$ & (YY) YTY .Y & $($ rI) Vrr.q & $(\text { F97-48c) })^{1 r}$ \\
\hline$(r+)^{10.10}$ & $(r Y)^{10.11}$ & $(r Y)^{19.0 V}$ & $(r+)^{\prime T} \cdot 11$ & $(19)^{110 V_{.}}$ & $(1 \wedge)^{\wedge r Y .4}$ & $(17)^{1} \leqslant 4 . r$ & $(1 \wedge)^{1 . v 1.1}$ & $(\text { F98-108c) })^{\top r}$ \\
\hline$(1 \Lambda)^{\prime V .} . \varepsilon \Lambda$ & (1Y) YV.\&Y & $(1 \Lambda)^{\prime \Lambda . V Y r}$ & $(I V)$ IV.ru & $(9)^{1} \leq 10.1$ & $(1 \cdot)^{1} \cdot \varepsilon 1.9$ & $(10) 101 \mathrm{~V} .1$ & (4) & $(F 85)^{\prime !}$ \\
\hline$(I r)^{\prime A} . Y \xi$ & $(14)^{1 \wedge .14}$ & $(1 Y)^{19 . \% 1}$ & $(14)^{18.91}$ & (IY) & (Ir) & $(1+)^{l o v} \cdot r$ & $(1+)^{\prime Y Y A V .}$. & $($ F97-113c) 10 \\
\hline$(r \cdot)^{\prime 4} \wedge . \wedge r$ & $(r \cdot)^{14.41}$ & $(r \cdot)^{\wedge \wedge .} \cdot r$ & $(19)^{14 . \Delta V}$ & $(0)^{101 \wedge . V}$ & $(0) 111 \% . V$ & $(v) 170 \leqslant .$. & $(r) 1 \leqslant 9 r .4$ & 191(أباء99) \\
\hline$(r)^{r} \cdot . r 4$ & $\left(r^{r}\right)^{r} . \xi 1$ & $(0)^{r} 1 . \leqslant 1$ & $(r)^{19.8 \%}$ & (1')14A4.0 & $(v) 1 \cdot v \varepsilon .$. & (11) 1094.0 & 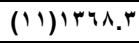 & 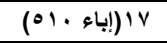 \\
\hline (1) r 1.9 \& & $(1)^{r} 1.04$ & (I)YY.YT & $(1 \cdot)^{1 \wedge .0 V}$ & $(1 \wedge)^{11 r \cdot .9}$ & $(17)^{\wedge \times 0.9}$ & $(19) \backslash \leqslant Y \leqslant . r$ & $(19) 1179.4$ & 1/ (دجلة) \\
\hline$(10)$ IV.VY & 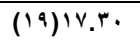 & $(r)$ IV.qu & $(10) 1 y . \leqslant 0$ & (I) IVYY. & (1) Irar.r & $(1)^{1 \wedge \wedge \wedge .0}$ & $(1 \cdot) 1 \leqslant r r .9$ & 99 \\
\hline$(r) 19.0$. & $(r) 18.4 \pi$ & $(I V)^{\prime A . V Y r}$ & $(r \cdot)^{14 . \cdot 1}$ & $(r)^{10 \leqslant \varepsilon}{ }^{\prime}$ & $(v)^{110 \leqslant .4}$ & (ग) ITAY.V & $(0) 1 \leqslant 70.1$ & . ب (محلي شوكي) \\
\hline$(11)^{\wedge \wedge .} \leq 0$ & $(11)^{1 \wedge .80}$ & $\left.(\wedge)^{r} \cdot.\right)^{r}$ & $(\wedge) \backslash \wedge . \vee \cdot$ & $(10) 11 V \leqslant . V$ & $(1 \varepsilon)^{99 \vee .4}$ & $(0) 1791 . \wedge$ & $(10) 11 \times 1.8$ & 1" (مراكثي) \\
\hline$(q)^{1 \wedge . \vee q}$ & $(\wedge)^{\prime \wedge . \vee \vee \Lambda}$ & $(v) r \cdot, r$. & $(0) \backslash \wedge . \wedge r$ & $(1 r) i r 01 . r$ & $(1 r)^{1} \ldots$. & $(\xi) \backslash V I \varepsilon . V$ & 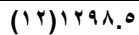 & r r (محلي) \\
\hline
\end{tabular}

\title{
GENETIC STABILITY FOR CULTIVARS AND LINES OF CHICPEA (Cicer arietinum L.) UNDER RAINFALL CONDITIONS IN NORTHERN OF IRAQ
}

Mohammed Y. H. Al-Fahady Maan M. Al-Badrany

Dept. of Felid crops , College of Agric. And Forestry, Mosul Uni., Iraq

\begin{abstract}
22 chickpea genotypes (cultivars and lines) were growing in five different environments under rainfed conditions in north Iraq(Mosul and Alkosh in season 2004-2005 and the same locations beside Al-Rashidia in season 2005-2006) to evaluate stability of seed yield, its components and protein content .Combined analysis showed a significant differences at $1 \%$ level for genotype, environments and their interactions for all characters. Local variety exhibited the highest stability order for no. of days to $50 \%$ flowering and $90 \%$ maturaty with four stability methods. Dijla variety showed the highest rank for plant height and protein content. Rafidain gave the highest order for no. of secondary branches, no. of pods per plant and seed yield $(\mathrm{kg} / \mathrm{ha})$ in most stability methods.
\end{abstract}

\section{المصادر}

Allard, R. W. and A. D. Bradshaw (1964). Implications of genotype-environment interaction in applied plant breeding. Crop Sci., $4: 503-507$.

Akhtar, L. H. ; Abdullah ; S. Z. Siddiqi ; M. Hussain and M. Arshad (2003). Evaluation of exotic material of chickpea (Cicer arietinum) under Bahawalpur agroclimatic condition. Asian J. of Plant Sci., 2 (4) : 406-408.

A.O.A.C. (1980) . Association of official Agriculture Chemists "Official Methods of Analysis" $13^{\text {th }}$ ed. Washington D.C., U.S.A. Cereal.Chem. $63: 191-193$. 


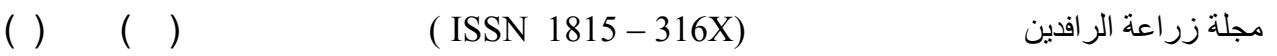

Bala, A. ; D. R. Satija ; S. K. Gupta and V. P. Gupta (1993) . Combining ability analysis for proteins and its fractions in chickpea. Crop Improvement, 20 (2): 207-210 .

Chander, S. ; R. Dhari and R. Kumar (2001) .Variation in selected recombinant inbred lines of two crosses in chickpea (Cicer arietinum L.) . Annals of Biology, 17 (1) : 29-34 .

Eberhart, S. A., and W. A. Russell. (1966). Stability parameters for comparing varieties . Crop Sci. $6: 36-40$.

Eskridge, K. M. (1990) . Selection of stable cultivars a safety-first rule . Crop Science, $30: 369-374$.

Finaly, K. W. and G. N. Wilkinson (1963). The analysis of adaptation in a plantbreeding programme . Australian J. Agric. Res. 14:742-754, Sited by Al-Haza'a (2001).

Gastel , A. J. G. ; Z. Bishaw and M. Diekmann (1994) . legume seed production . Hand out for course - Iraq, seed unit, ICARDA, in 22 Jun -3 July .

Kataoka, S. (1963) A stochastic programming model . Econometrica 31: 181-196 . (Crop Sci. 30: 369-347. 1990).

Katiyar, M. (2003) .Genetic analysis of yield and its component traits in kabuli chickpea. Indian Journal of Pulses Research 16(2) : 92-94 .

Kumar, S. ; H. A. Van Rheenen and O. Singh (1999) . Genetic analysis of different components of crop duration in chickpea. Journal of Genetics and Breeding . 53 (3) : 189-200 .

Malhotra, R. S. ; K. B. Singh and M. S. Saxena (1997). Effect of irrigation on winter sown chickpea in Mediterranean environment . Agronomy and Crop Science $178: 237-243$.

Mehla, I. S. ; R. S. Waldia and S. S. Dahiya (2000) Phenotypic stability for some cooking quality attributes among kabuli chickpea (Cicer arietinum L.) genotypes. J. Genet. \& Breed., 54: 293-297.

Miller , P. A. ; H. F. Robinson and O. H. Pope (1962) . Cotton variety testing : Additional information on variety x environmental . Crop Sci. 2 : 349-352.

Ozgun, O. S. ; B. T. Bicer and D. Sakar (2004) Agronomic and morphological characters of chickpea under irrigated conditions in Turkey . Int. J. Agric. Biol., 6 (4) : 606-610.

Pundir, R. P. S. and G. V. Reddy (1998) . Two new traits open flower and small leaf in chickpea (Cicer arietinum L.). Euphytica, 102: 357-361.

Singh, K.B. ; R. S. Malhotra ; M. C. Saxena and G. Bejiga (1997) . Superiority of Winter Sowing over traditional spring sowing of chickpea in the Mediterranean Region . Agron. J., 89 : 112-118 .

Singh, K. B. ; P. C. Williams and H. Nakkoul (1990) . Influence of growing season, location and planting time of some quality parameters of kabuli chickpea. J. Sci. Food Agric. ,53: 429-441 .

Sharif, A. ; A. Bakhsh ; M. Arshad ; A. M. Haqqani and S. Najma (2001) Identification of genetically superior hybrids in chickpea (Cicer arietinum L.).Pakistan Journal of Botany 33 (4) : 403-4090.

Shukla, G. K. (1972) . Some statistical aspects of partitioning genotype environmental component of variance. Heredity $29: 237-245$.

Yates, F., and W. G. Cochran. (1938) . The analysis of groups of experiments. J. Agric. Sci. 28 : 556-580. 